\title{
Effect of a Whole-Body Vibration Training Modifying the Training Frequency of Workouts Per Week in Active Adults
}

\author{
Esmeraldo Martínez-Pardo, ${ }^{1,2}$ Salvador Romero-Arenas, ${ }^{1,2}$ Enrique Martínez-Ruiz,, 3 \\ Jacobo A. Rubio-Arias, ${ }^{1,2}$ and Pedro E. Alcaraz ${ }^{1,2}$ \\ ${ }^{1}$ Research Center for High Performance Sport-UCAM, Catholic University of Murcia, Murcia, España; ${ }^{2}$ Faculty of Sport- \\ UCAM, Catholic University of Murcia, Murcia, Espana; and ${ }^{3}$ Faculty of Health Sciences, Catholic University of Murcia, \\ Murcia, Spain
}

\begin{abstract}
Martínez-Pardo, E, Romero-Arenas, S, Martínez-Ruiz, E, Rubio-arias, JA, and Alcaraz, PE. Effect of a whole-body vibration training modifying the training frequency of workouts per week in active adults. $J$ Strength Cond Res 28(11): 3255$3263,2014-$ The aim of this study was to evaluate the effects of whole-body vibration by varying the training frequency ( 2 or 3 sessions per week) on the development of strength, body composition, and mechanical power. Forty-one (32 men and 9 women) recreationally active subjects $(21.4 \pm 3.0$ years old; $172.6 \pm 10.9 \mathrm{~cm} ; 70.9 \pm 12.3 \mathrm{~kg}$ ) took part in the study divided in 2 experimental groups ( $\mathrm{G} 2=2$ sessions per week, $\mathrm{G} 3=3$ sessions per week) and a control group (CG). The frequency of vibration $(50 \mathrm{~Hz})$, amplitude $(4 \mathrm{~mm})$, time of work (60 seconds), and time of rest (60 seconds) were constant for G2 and G3 groups. Maximum isokinetic strength, body composition, and performance in vertical jumps were evaluated at the beginning and the end of the training cycle. A statistically significant increase of isokinetic strength was observed in G2 and G3 at angular velocities of 60, 180, and $270^{\circ} \cdot \mathrm{s}^{-1}$. Total fat-free mass was statistically significantly increased in G2 $(0.9 \pm 1.0 \mathrm{~kg})$ and G3 $(1.5 \pm 0.7$ $\mathrm{kg})$. In addition, statistically significant differences between G3 and CG $(1.04 \pm 1.7 \%)(p=0.05)$ were found. There were no statistically significant changes in the total fat mass, fat percentage, bone mineral content, and bone mineral density in any of the groups. Both vibration training schedules produced statistically significant improvements in isokinetic strength. The vibration magnitude of the study presented an adaptation stimulus for muscle hypertrophy. The vibration
\end{abstract}

Address correspondence to Pedro E. Alcaraz, palcaraz@ucam.edu.

28(11)/3255-3263

Journal of Strength and Conditioning Research

(c) 2014 National Strength and Conditioning Association training used in this study may be valid for athletes to develop both strength and hypertrophy of the lower limbs.

KEY WoRDS vibration platform, strength, power, body composition

\section{INTRODUCTION}

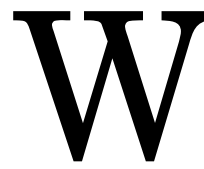

hole-body vibration (WBV) has been suggested as a training and rehabilitation method (41). In sport practice, vibration is applied to the entire limb, to the entire body (26), through a vibrating platform on which a person stands for a certain period of time (11). The exercise devices currently available on the market deliver vibration to the whole body by means of oscillating plates using 2 different systems: (a) reciprocating vertical displacements on the left and right side of a fulcrum and (b) the whole plate oscillating uniformly up and down (10). In most devices, such vibratory movements generate sinusoidal oscillations that are characterized by their amplitude (in millimeter) and frequency (in hertz). During vibration, the human body is accelerated by causing a reactive force by and within the human body (42).

The reported benefits of vibration include improvements in bone health and neuromuscular function (50). The effects of vibration exposure have been examined using different protocols and methods. Different studies on vibration platforms have shown significant improvements in muscle strength and power in different populations $(5,6)$. Some studies also suggest that WBV might affect cardiovascular responses during exercise $(23,43), \dot{\mathrm{V}}_{2}$ kinetics (30), and produces significant increase in plasma concentrations of testosterone and growth hormone, modifying the activity of the endocrine system (6).

The main mechanisms explaining the increase in muscle strength derived from vibration training are neural regulation of voluntary muscle contraction and the neuromuscular adaptations that occur (26). A previous study showed that $24 \mathrm{WBV}$ sessions over 8 weeks $(30 \mathrm{~Hz} ; 5 \mathrm{~mm})$ is an effective 
TABLE 1. Characteristics of the participants. *

\begin{tabular}{|c|c|c|c|c|}
\hline & Age (y) & Height $(\mathrm{cm})$ & Body mass $(\mathrm{kg})$ & $\begin{array}{c}\text { TorqF } 270^{\circ} \cdot \mathrm{s}^{-1} \\
(\mathrm{~N} \cdot \mathrm{m})\end{array}$ \\
\hline $\mathrm{G} 2(n=16)$ & $21.5 \pm 5.2$ & $175.1 \pm 8.1$ & $71.2 \pm 12.6$ & $124.8 \pm 37.8$ \\
\hline G3 $(n=14)$ & $21.1 \pm 1.6$ & $170.3 \pm 15.8$ & $74.8 \pm 13.9$ & $123.7 \pm 39.9$ \\
\hline CG $(n=11)$ & $21.5 \pm 3.8$ & $172.3 \pm 8.9$ & $66.8 \pm 10.5$ & $132.0 \pm 33.3$ \\
\hline Total $(n=41)$ & $21.4 \pm 3.0$ & $172.6 \pm 10.9$ & $70.9 \pm 12.3$ & $126.8 \pm 37$ \\
\hline
\end{tabular}

${ }^{*}$ TorqF $=$ peak torque in knee extension; $\mathrm{G} 2=2$ days training group; $\mathrm{G} 3=3$ days training group; $\mathrm{CG}=$ control group.
Reduction of body fat and the increase of muscle mass are some of the most popular objectives for starting an exercise program (44). However, there are few studies that analyze the effects of WBV training on body composition. For example, a recent study has found that high amplitude WBV training can elicit lean body mass in healthy students (45). Another study (23) found that there was a reciprocal increase $(+2.2 \%)$ in fat-free mass in

training methodology for inducing improvements in kneeextensor explosiveness (3). When the effects of WBV were evaluated on vertical jumps, improvements in the performance of the squat jump (SJ) (9) and countermovement jump (CMJ) $(3,46)$ were found. Torvinen et al. (48) suggest that short-time (4 minutes) exposure to WBV can lead to an improvement in countermovement vertical jump performance and force generating capacity in maximal isometric strength of the leg extensors. Similar results were found by Cardinale (9) after 10 days of vibration exercise involving male athletes and reported a considerable improvement in vertical jumping (CMJ and 5 seconds of continuous jumping) and muscular strength (maximal dynamic leg press exercises on a slide machine with extra loads of 70, 90, 110, and $130 \mathrm{~kg}$ ). Apparently, this increased muscle activity could be due to vibration signals that activate muscle spindle receptors, which in turn causes reflexive activation of motor units (45). Furthermore, Lamont et al. (30) have shown that 6 weeks of training seems to have been sufficient to have produced statistically significant improvements in power measures like jump height (in centimeters) and peak power (Pmax) for SJs. In contrast, de Ruiter et al. (16) found that 11 weeks of standard 2-legged WBV training $(30 \mathrm{~Hz} ; 8 \mathrm{~mm})$ without additional training loads did not improve functional knee extensor muscle strength (CMJs) in 10 young healthy physically active subjects.

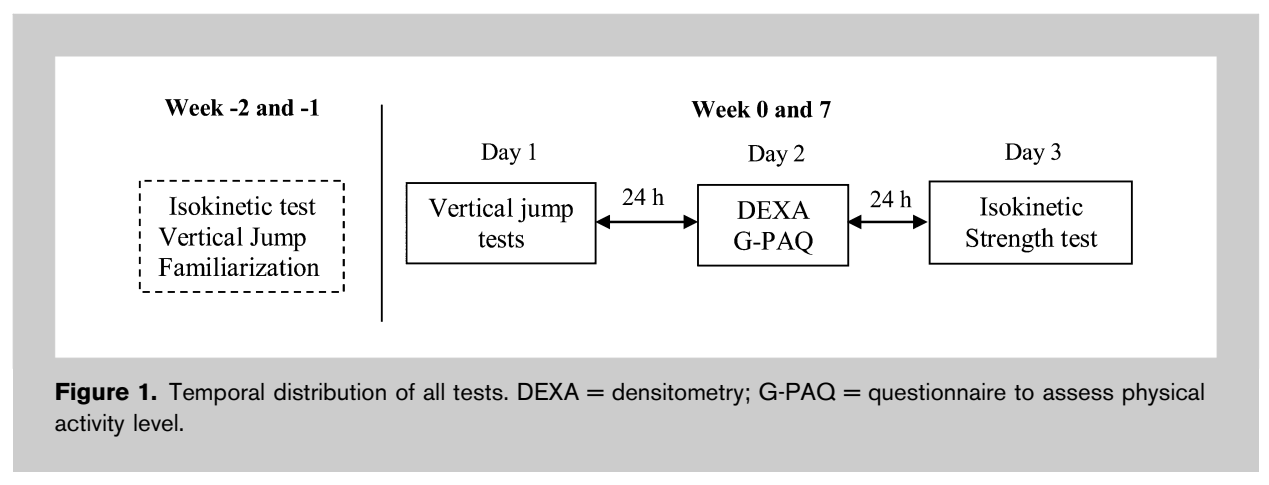
untrained young women after 24 weeks of WBV training. The WBV group trained 3 times weekly increasing training volume (35-40 Hz; $2.5-5 \mathrm{~mm}$; 3-20 minutes; number of series; shortening rest periods). However, there were no changes in total fat (44). Similar results were found by Fjeldstad et al. (20) who demonstrated that training with WBV with progressive overload $(30-40 \mathrm{~Hz} ; 3 \mathrm{~mm})$ plus resistance training 3 times per week for 8 months resulted in positive body composition changes of increased lean tissue in older women (20). Also, a preliminary study done by Vissers et al. (52) shows how 6 months of WBV training (30$40 \mathrm{~Hz}$; low-high amplitude; 10-22 exercises) may influence reduction in visceral fat of obese adults $\left(3 \mathrm{~d} \cdot \mathrm{wk}^{-1}\right)$. However, Verschueren et al. (50) found that muscle mass was not affected in postmenopausal women after 6 months of WBV training ( 3 sessions per week) increasing systematically the intensity $(35-40 \mathrm{~Hz} ; 1.7-2.5 \mathrm{~mm}$ ) and the volume (duration of 1 vibration session; number of series of 1 exercise; number of different exercises). Therefore, further research is necessary to clarify the frequency of training sessions per week.

Training adaptations are determined by various factors (2), one of those factors is the optimal training frequency (the number of workouts per week). The frequency of training depends on the number of muscle groups trained per workout as well as the volume and intensity (2). Frequencies of 2-3 days per week have been effective in 29 untrained volunteers (8). A meta-analysis study has shown that strength gains in untrained individuals were highest with a frequency of 3 days per week (40). However, there are no studies that compare different training frequencies in $\mathrm{WBV}$ training. Therefore, the aim of this research was to study the effects, when using WBV with 2 different training frequencies ( 2 vs. $3 \mathrm{~d} \cdot \mathrm{wk}^{-1}$ ), on the development of strength, mechanical power of the muscles of 
TABLE 2. Weekly distribution of the parameters of vibration training.*

\begin{tabular}{lllllll}
\hline & W1 & \multicolumn{1}{c}{ W2 } & \multicolumn{1}{c}{ W3 } & \multicolumn{1}{c}{ W4 } & \multicolumn{1}{c}{ W5 } & \multicolumn{1}{c}{ W6 } \\
\hline G2 & $8 \times 60 \mathrm{~s}$ & $9 \times 60 \mathrm{~s}$ & $10 \times 60 \mathrm{~s}$ & $11 \times 60 \mathrm{~s}$ & $12 \times 60 \mathrm{~s}$ & $13 \times 60 \mathrm{~s}$ \\
& $4 \mathrm{~mm}$ & $4 \mathrm{~mm}$ & $4 \mathrm{~mm}$ & $4 \mathrm{~mm}$ & $4 \mathrm{~mm}$ & $4 \mathrm{~mm}$ \\
G3 & $8 \times 60 \mathrm{~s}$ & $9 \times 60 \mathrm{~s}$ & $10 \times 60 \mathrm{~s}$ & $11 \times 60 \mathrm{~s}$ & $12 \times 60 \mathrm{~s}$ & $13 \times 60 \mathrm{~s}$ \\
& $4 \mathrm{~mm}$ & $4 \mathrm{~mm}$ & $4 \mathrm{~mm}$ & $4 \mathrm{~mm}$ & $4 \mathrm{~mm}$ & $4 \mathrm{~mm}$ \\
CG & No vibration training & & & & \\
\hline
\end{tabular}

$* \mathrm{~W}=$ week; $\mathrm{G} 2=2$ days training group; $\mathrm{G} 3=3$ days training group; $\mathrm{CG}=$ control group. habitual physical activity level measured with the G-PAQ questionnaire, sex, and isokinetic strength of the knee extensor (Table 1), and then assigned to 1 of 2 treatment conditions, or the CG: (a) G2 $=2 \mathrm{WBV}$ training per week, (b) G3 $=3$ WBV training session per week, and (c) a no training CG. Subjects completed 1 week of familiarization WBV training before a 6-week specific training phase. During the 1-week familiarizathe lower limbs, and changes in body composition in active adults. Additionally, the following hypothesis was established: the WBV training program, using 3 days per week will produce an increase in mechanical power, strength, and muscle mass and decrease fat mass in young healthy adults, whereas the 2 days of training will not.

\section{Methods}

\section{Experimental Approach to the Problem}

A quasi-experimental pretest/posttest group design using 2 training groups and a control group $(\mathrm{CG})$ was used to examine the short-term effects of 2 vs. 3 sessions per week when using WBV on the development of lower-body strength, mechanical power, and body composition. Before data collection, the participants took part in a familiarization session for each test. To reduce potential confounding, a matched design was used in which participants were matched depending on their

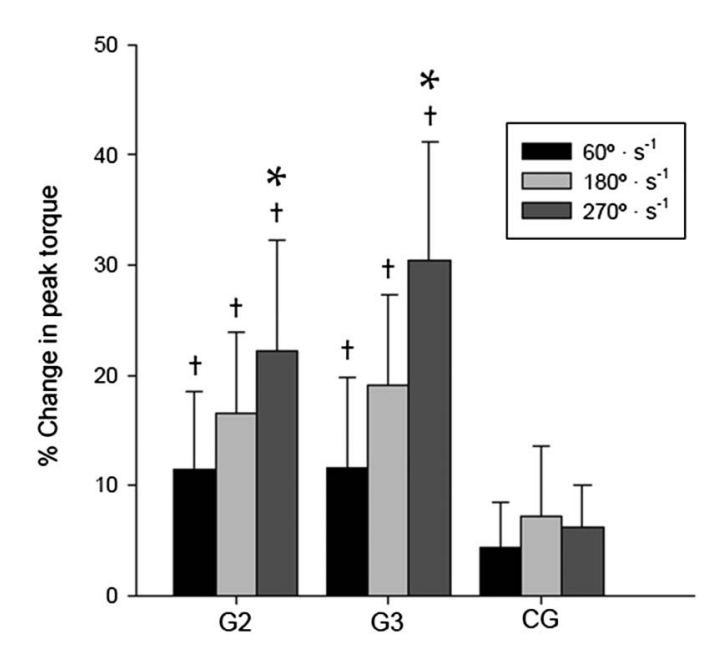

Figure 2. Changes in isokinetic peak torque for knee extensors at angular velocities of 60,180 , and $270^{\circ} \cdot \mathrm{s}^{-1} \cdot \mathrm{G} 2=2$ days training group; G3 $=3$ days training group; $\mathrm{CG}=$ control group; $\dagger=$ statistically significant differences $(p \leq 0.05)$ between pretest and posttest; ${ }^{*}=$ statistically significant differences $(p \leq 0.05)$ with the CG. tion phase, subjects performed low-load magnitude WBV training; additionally, participants were familiarized with the measurement protocols (vertical jump and isokinetic tests).

\section{Subjects}

Forty-one recreationally active students $(n=41 ; 32$ men and 9 women; $21.4 \pm 3.0$ years old range $19-32 ; 172.6 \pm$ $10.9 \mathrm{~cm} ; 70.9 \pm 12.3 \mathrm{~kg}$ ) took part in the study (Table 1 ). Recreationally active were classified as engaging in low-tomoderate intensity physical activity no more than 3 times per week for approximately 20-30 minutes. Each subject read and signed a University Institutional Review Board approved informed consent form before participation.

\section{Testing}

The initial and final assessment was carried out at the beginning and end of the experimental phase. One week was used to accomplish the tests. Participants performed the initial and final test in the same sequence and at the same time of day (Figure 1). Two weeks before the initial data collection, 2 familiarization sessions were implemented for the jumps test. All tests involving muscle actions were performed with a rest of 48 hours between each measurement session, with the aim of ensuring that the participants were not suffering from fatigue when they had to perform.

\section{Jump Procedures} platform (Dinascan/IBV, Valencia, Spain). In all jump tests, the subjects were instructed to keep their hands on their waist at all times to minimize any contribution to jump impulse by the upper body (13). Each subject performed a practice trial for each of the movements before performing the test trials. All variables of the jump tests were taken in absolute terms and relative to body mass $(\mathrm{Bm})(4)$. The SJs were performed starting from a $90^{\circ}$ knee angle position, and no drop or countermovement was permitted. If any countermovement was detected on the force-time display, the subject was required to repeat that trial. For the CMJs, the subjects were instructed to perform the jump as fast as possible with the aim that the stretch-shortening cycle be activated (28). The force-time traces for the SJs and CMJs were
Jump tests (SJ and CMJ) were performed on a force 
TABLE 3. Body composition variables (mean $\pm S D)$.

\begin{tabular}{lccccc}
\hline & & & & \multicolumn{1}{c}{$\begin{array}{l}\mathrm{BMD}_{\text {total }} \\
\left(\mathrm{g} \cdot \mathrm{cm}^{-2}\right)\end{array}$} & $\mathrm{BMC}_{\text {total }}(\mathrm{g})$ \\
\hline G2 Pre & $18.3 \pm 6.8$ & $54.7 \pm 10.8$ & $13.1 \pm 5.9$ & $1.1 \pm 0.1$ & $3,106 \pm 445$ \\
Post & $17.6 \pm 7.7$ & $55.6 \pm 10.9 \dagger$ & $12.6 \pm 6.9$ & $1.1 \pm 0.1$ & $3,104 \pm 435$ \\
$\Delta$ & $-0.8 \pm 1.7$ & $0.9 \pm 1.0$ & $-0.5 \pm 1.4$ & $0.0 \pm 0.0$ & $-1.1 \pm 21.9$ \\
G3 Pre & $20.7 \pm 7.3$ & $53.7 \pm 10.5$ & $14.6 \pm 4.9$ & $1.1 \pm 0.1$ & $3,035 \pm 319$ \\
Post & $19.8 \pm 7.4$ & $55.1 \pm 10.4 \dagger$ & $14.2 \pm 5.8$ & $1.1 \pm 0.1$ & $3,036 \pm 326$ \\
$\Delta$ & $-0.8 \pm 1.9$ & $1.5 \pm 0.7$ & $-0.4 \pm 1.3$ & $0.0 \pm 0.0$ & $0.9 \pm 27.6$ \\
CG Pre & $20.6 \pm 7.6$ & $49.4 \pm 10.8$ & $13.1 \pm 4.1$ & $1.1 \pm 0.2$ & $2,968 \pm 542$ \\
Post & $21.1 \pm 7.3$ & $49.7 \pm 10.7$ & $13.8 \pm 3.9$ & $1.1 \pm 0.2$ & $2,971 \pm 550$ \\
$\Delta$ & $0.6 \pm 1.9$ & $0.4 \pm 0.7 \ddagger$ & $0.7 \pm 1.4$ & $0.0 \pm 0.0$ & $3.3 \pm 22.8$ \\
\hline
\end{tabular}

${ }^{*} \mathrm{G} 2=2$ days training group; $\mathrm{G} 3=3$ days training group; $\mathrm{CG}=$ control group; $F M=$ fat mass, $\mathrm{FFM}=$ fat-free mass; $\mathrm{BMD}=$ bone mineral density; $\mathrm{BMC}=$ bone mineral content; $\Delta=$ difference.

Statistically significant difference $(p \leq 0.05)$ between pretest and posttest.

$\ddagger$ Statistically significant difference $(p \leq 0.05)$ with G3.

strength tests. Each subject underwent a thorough and standardized familiarization session, which included all tests, at least 1 week before being tested. The hip extensors and flexors in the dominant leg were tested concentrically. All movements were tested at 60,180 , and $270^{\circ} \cdot \mathrm{s}^{-1}$ angular velocities. Each subject was measured in a standing position and stabilized with velcro straps. The axis of rotation of the dynamometer lever arm was aligned with the anatomical axis of the hip, as described in the Biodex test manual. The dynamometer was calibrated before each test session, and a gravitational cor-

analyzed to obtain 3 dependent variables namely: jump height, maximum mechanical power (Pmax), and the maximum rate of force development (RFDmax). The start of concentric contraction was defined as the point where the force readings were $10 \mathrm{~N}$ greater than the average of the force readings when the subject was static in the SJ starting position. Jump heights $(h)$ were calculated from the take off vertical velocity $(v)$ using the following equation: $h=v^{2} \cdot 2 g^{-1}$. Absolute and relative mechanical power were calculated as follows: vertical force $\times$ instantaneous vertical velocity of the system's center of mass (12), and RFDmax was calculated as the greatest rise in force during 5-millisecond periods from the start of the concentric contraction (23).

\section{Isokinetic Strength}

An isokinetic dynamometer (Biodex System 3; Biodex Medical Systems Inc., Shirley, NY, USA) was used for the isokinetic rection was calculated by the dynamometer and automatically compensated for the measurements.

At each test velocity, the subject performed 5 submaximal warm-up trials followed by 3 maximal warm-up trials. The test started 1 minute after the 6 warm-up trials had been completed. A recovery period of 90 seconds (7) between test velocities was used. After the warm-up trials, 3 maximal trials were performed for each test (1). The trial in each test which had the greatest peak torque was taken as the measure of maximal strength. Results were normalized, expressed relative to body mass.

\section{Body Composition}

Total and regional bone, fat, and fat-free masses were assessed by DEXA (XR-46; Norland Corp., Fort Atkinson, WI, USA). The DEXA scanner was calibrated using a lumbar spine phantom as recommended by the manufacturer. Subjects were scanned in the supine position. Fat-free mass (in grams), fat mass (in grams), total area (square centimeter), and bone mineral content (BMC) (in grams) were calculated from total and regional analysis of the whole-body scan. Areal bone mineral density (BMD, in grams per square centimeter) was calculated using the formula BMD = $\operatorname{BMC}(\mathrm{g}) \times$ area $\left(\mathrm{cm}^{2}\right)^{-1}$.

\section{Vibration Protocol}

The vibration stimulus consisted of uniform vertical oscillations Power PlateNext Generation (Power Plate North America, Northbrook, IL, USA). Subjects stood on the platform 
TABLE 5. Peak mechanical power in SJ and CMJ, absolute and relative to body mass (mean $\pm S D)$.*

\begin{tabular}{llccc}
\hline & $S J_{P \max }(\mathrm{W})$ & $C M J_{\text {Pmax }}(\mathrm{W})$ & $\begin{array}{c}S J_{\mathrm{Pmax} / \mathrm{bm}} \\
\left(\mathrm{W} \cdot \mathrm{kgg}^{-1}\right)\end{array}$ & $\begin{array}{c}\mathrm{CM} J_{P \max / \mathrm{bm}} \\
\left(\mathrm{W} \cdot \mathrm{kgf}^{-1}\right)\end{array}$ \\
\hline G2 Pre & $3,518 \pm 868$ & $3,177 \pm 736$ & $48.9 \pm 6.2$ & $44.4 \pm 6.2$ \\
Post & $3,382 \pm 847$ & $3,155 \pm 765$ & $48.1 \pm 15.4$ & $43.7 \pm 5.7$ \\
$\Delta$ & $-136.2 \pm 379.9$ & $-21.1 \pm 225.7$ & $-0.8 \pm 13.0$ & $-0.7 \pm 3.3$ \\
G3 Pre & $3,497 \pm 763$ & $3,261 \pm 658$ & $48.0 \pm 6.5$ & $44.8 \pm 5.5$ \\
Post & $3,409 \pm 737$ & $3,281 \pm 705$ & $51.1 \pm 13.2$ & $44.2 \pm 4.7$ \\
$\Delta$ & $-87.8 \pm 254.9$ & $20.2 \pm 373.1$ & $3.1 \pm 12.2$ & $-0.6 \pm 5.6$ \\
CG Pre & $3,207 \pm 743$ & $2,928 \pm 610$ & $47.8 \pm 6.2$ & $43.9 \pm 4.4$ \\
Post & $3,373 \pm 743$ & $2,946 \pm 609$ & $52.2 \pm 11.4$ & $43.8 \pm 3.9$ \\
$\Delta$ & $165.9 \pm 290.4$ & $18.2 \pm 107.9$ & $4.4 \pm 9.7$ & $-0.1 \pm 2.3$ \\
\hline
\end{tabular}

${ }^{*} \mathrm{G} 2=2$ days training group; $\mathrm{G} 3=3$ days training group; $\mathrm{CG}=$ control group; $\mathrm{S} \mathrm{J}=$ jump without countermovement; $\mathrm{CMJ}=$ countermovement jump; MIP = maximum instantaneous power; $\mathrm{bm}=$ body mass; $\Delta=$ difference.

\section{Statistical Analyses}

Data were stored using the spreadsheet Excel 2003 (Microsoft Corp., Redmond, WA, USA). Statistical analyses of data were performed with SPSS 15.0 (SPSS 15.0; SPSS, Inc., Chicago, IL, USA) in the Windows environment. A descriptive analysis was performed to detail and analyze the characteristics of the sample participating in the study.

For the inferential analysis, we performed the ShapiroWilks test to establish the normality of the sampling distribution and analysis of runs to observe the independence of observations. To determine the effect of independent variables

holding an isometric quarter squat position with the feet shoulder-width apart (32). After the familiarization week, subjects trained 2 or 3 days per week for 6 weeks (with the exception of the $\mathrm{CG}$ ) using a vibrating incremental training program that began with 8 sets per session and increasing by 1 set weekly maintaining a series of parameters: vibration frequency $(50 \mathrm{~Hz})$, vibration amplitude $(4 \mathrm{~mm})$, working time (60 seconds), and recovery time (60 seconds) constant for the 2 groups (G2 $=2$ days, G3 $=3$ days). The International Society of Musculoskeletal and Neuronal Interactions has been taken into consideration for the design of this protocol (38) (Table 2).

TABLE 6. Maximum rate of force development in the SJ and CMJ.*

\begin{tabular}{llcc}
\hline & \multicolumn{1}{c}{$\begin{array}{c}S J_{\text {RFDmax }} \\
\left(\mathrm{N} \cdot \mathrm{s}^{-1}\right)\end{array}$} & $\begin{array}{c}C M J_{\text {RFDmax }} \\
\left(\mathrm{N} \cdot \mathrm{s}^{-1}\right)\end{array}$ \\
\hline G2 & Pre & $1,055 \pm 506$ & $1,243 \pm 544$ \\
& Post & $1,316 \pm 693$ & $1,313 \pm 562$ \\
& $\Delta$ & $161.1 \pm 393.6$ & $70.2 \pm 733.0$ \\
G3 & Pre & $861 \pm 365$ & $1,083 \pm 294$ \\
& Post & $1,074 \pm 424$ & $1,279 \pm 434$ \\
& $\Delta$ & $213.4 \pm 402.1$ & $196.5 \pm 434.5$ \\
CG & Pre & $936 \pm 349$ & $1,197 \pm 365$ \\
& Post & $1,116.0 \pm 343.0$ & $1,260.0 \pm 543.0$ \\
& $\Delta$ & $179.8 \pm 375.6$ & $62.7 \pm 486.8$ \\
\hline
\end{tabular}

${ }^{*} \mathrm{G} 2=2$ days training group; $\mathrm{G} 3=3$ days training group; $\mathrm{CG}=$ control group; $\mathrm{SJ}=$ squat jump; $\mathrm{CMJ}=$ countermovement jump; RFDmax $=$ ratio of maximum development of strength; $\Delta=$ difference. on the dependent variable, a repeated measures analysis of variance (ANOVA) was carried out for the entire sample. If there were statistically significant differences $(p \leq 0.05)$ for the time factor, a repeated measures ANOVA test (General Linear Model) was performed to assess repeated measures of each group to differentiate between pretest and posttest sessions. If there were statistically significant differences $(p \leq 0.05)$ for the time $\times$ group factor ANOVA, a Tukey's post hoc test was performed.

\section{Results}

This study was designed to investigate the effects of 6 weeks of training with body vibrations modifying the training days. Below show the results for isokinetic strength, vertical jump, and body composition measurements of the groups over the study period.

\section{Isokinetic Strength}

Figure 2 presents relative gains in peak torque between the pretest and posttest for each group: G2 $\left(60^{\circ} \cdot \mathrm{s}^{-1}=\right.$ $11.5 \pm 14.0 \%, 180^{\circ} \cdot \mathrm{s}^{-1}=16.5 \pm 14.7 \%, 270^{\circ} \cdot \mathrm{s}^{-1}=22.1 \pm$ $20.3 \%)$, G3 $\left(60^{\circ} \cdot \mathrm{s}^{-1}=11.6 \pm 16.5 \%, 180^{\circ} \cdot \mathrm{s}^{-1}=19.1 \pm 16.5 \%\right.$, $\left.270^{\circ} \cdot \mathrm{s}^{-1}=30.5 \pm 21.5 \%\right)$, and CG $\left(60^{\circ} \cdot \mathrm{s}^{-1}=4.4 \pm 8.1 \%\right.$; $\left.180^{\circ} \cdot \mathrm{s}^{-1}=7.2 \pm 12.7 \%, 270^{\circ} \cdot \mathrm{s}^{-1}=6.2 \pm 7.6 \%\right)$. Statistically significant differences were observed when comparing the effect of time on the experimental groups with CG. Statistically significant differences were found between G3 and CG $(p=0.066)$ at angular velocities of $180^{\circ} \cdot \mathrm{s}^{-1}$. In turn, at angular velocities of $270^{\circ} \cdot \mathrm{s}^{-1}$ were statistically significant differences between G2 $(p=0.041)$ and G3 $(p=0.001)$ compared with CG.

\section{Body Composition}

Table 3 shows the results of body composition variables for the experimental and CGs in the pretest posttest and 
changes (mean $\pm S D$ ). Statistically significant differences were observed $(p \leq 0.05)$ between pretest and posttest on fat-free mass for G2 $(p=0.005)$ and G3 $(p=0.001)$. Intergroups statistically significant differences were found between $\mathrm{G} 3$ and $C G$ in fat-free mass.

\section{Vertical Jump Performance}

Table 4 shows the results of the height of SJ and CMJ jumps, and the results of the height of these vertical jumps relative to $\mathrm{Bm}$ for the experimental groups and CGs in the pretest and posttest, and the changes (mean $\pm S D$ ). After a repeated measures ANOVA, no statistically significant changes were found $(p \leq 0.05)$ between pretest and posttest of any of the groups under study. $\mathrm{SJ}_{h}(p=0.112), \mathrm{CMJ}_{h}(p=0.646), \mathrm{SJ}_{\mathrm{h} / \mathrm{bm}}$ $(p=0.473)$, and $\mathrm{CMJ}_{\mathrm{h} / \mathrm{bm}}(p=0.254)$.

The results of absolute and relative peak mechanical power when performing a vertical jump (SJ and CMJ) of each participant can be seen in Table 5. By applying a repeated measures ANOVA, no statistically significant changes were observed $(p>0.05)$ in peak power developed during the $\mathrm{SJ}(p=0.689)$ nor during the $\mathrm{CMJ}(p=0.542)$ for any of the groups under a study of the pretest and posttest. There were not statistically significant changes $(p \leq 0.05)$ in peak power relative to $\mathrm{Bm}$ during the $\mathrm{SJ}(p=0.423)$ nor during the $\mathrm{CMJ}(p=0.833)$ in the experimental groups and the CG between pretest and posttest.

Table 6 shows the RFD obtained when performing the vertical jumps (SJ and $\mathrm{CMJ}$ ) with the experimental groups and CG in the pretest and posttest and the difference (mean $\pm S D$ ). No statistically significant differences between pretest and posttest in SJ and CMJ were found for any of the groups under study, nor were significant changes observed between groups for any vertical jumps studied.

\section{Discussion}

The aim of this study was to compare the effect of 6 weeks of WBV varying the weekly frequency of training (2 vs. 3 days) on body composition and lower limb isokinetic strength and power. An important finding from this study was the increased strength of knee extensor muscles for groups that were subjected to vibration. Furthermore, there was significant gain in the total fat-free mass in G2 $(0.9 \pm 1.0 \mathrm{~kg})$ and G3 $(1.5 \pm 0.7 \mathrm{~kg})$, establishing statistically significant differences between G3 and CG $(1.04 \pm 1.7 \%)(p=0.05)$. However, no significant changes in vertical jumping performance were found.

In the assessment of body composition, we found no statistically significant difference in the change in fat mass, $\mathrm{BMC}$, and $\mathrm{BMD}$ after vibration training. The opposite was true with the fat-free mass increased significantly in G2 and G3 between pretest and posttest. Furthermore, G3 statistically significant increased fat-free mass compared with CG. These results are similar to those reported by Hazell et al. (24), who claimed that the cardiovascular stress produced by exposure to $\mathrm{WBV}$ is moderate, and that the energy requirements could be compared with walking at a moderate intensity $(15,43)$. In addition, the total duration of the longer WBV session to participants who underwent the present work was 13 minutes. This is a too brief to produce changes in body fat. In a review, Rittweger (42), states that a person of $70 \mathrm{~kg}$, while performing WBV, consumes about $20 \mathrm{~L}$ of oxygen per hour. Assuming an energy equivalent of $20.9 \mathrm{~kJ} \cdot \mathrm{L}^{-1}$ of oxygen and caloric equivalent of $39 \mathrm{~kJ} \cdot \mathrm{g}^{-1}$ of fat, this would imply a loss of weight of only $10 \mathrm{~g}$ of fat for each hour of such exercise. Thus, WBV does not produce a minimum stimulus to generate loss of body fat with a 6-week training protocol.

There have been several studies suggesting that muscle hypertrophy may be due to a hormonal response induced by training (51). Such hormonal responses have been documented by an increase of testosterone (6), growth hormone $(6,29)$, increased catecholamine (22), decreased cortisol $(6,29)$, and increased protein synthesis (53). The literature shows that there is a greater acute increase in the production of growth hormone with exposure to vibration (6). These endocrine effects could be 1 explanation for the increase in fat-free mass after vibration training. In the Martinez-Pardo et al. (36) study, it was evident that 6 weeks of training using a high-amplitude vertical vibration platform, produced muscle hypertrophy in active subjects.

Regarding fat mass, there were no statistically significant changes in absolute or relative fat mass after 6 weeks of vibration training. There were also no significant differences when comparing the different groups together. This study provides results similar to those published by Roelants et al. (44), who assessed the body composition of 48 women with age and physical activity level similar to this study's participants. After 24 weeks of WBV, 3 sessions per week, with a frequency, amplitude, and time of exposure to vibration similar to this study, there were no changes in body fat. Comparing these results with those of the study by Roelants et al. (44), similar results were obtained. The authors assessed the body composition of 48 young women after 24-week vibration training, obtaining a significant increase of $2.2 \%$ fat-free mass between pretest and posttest. In this study, there was an increase of $1.6 \%$ in the G2 and 3\% in G3. The study by Roelants et al. (44) showed no reduction in body weight, total body fat, or subcutaneous fat after 24-week WBV training in previously untrained females. However, the results clearly showed that WBV training induced a gain in knee-extensor strength attended by small increases in fat-free mass. In this sense, we found a statistically significant increase in fat-free mass in 6 weeks. The participants of Roelants et al. (44) study were young women, however, there was a greater proportion of young men, who produce higher levels of testosterone. As mentioned above, there have been several studies suggesting that muscle hypertrophy may be due to a hormonal response induced by training (51), therefore, this may be the reason for increased fat-free mass. 
We report that a 6 -week WBV training program performed 2 or 3 days per week has not improved BMC nor BMD. Improvements did not occur when comparing each group being studied. However, Ligouri et al. (33) have found greater changes $(2.7 \%$ advantage over control) in BMD at the spine in 12 weeks. Furthermore, Gilsanz et al. (21) found improvements in trabecular BMD at the spine and cortical bone area of the femur in female participants after 12 months of WBV. But, there were no improvements in the randomized controlled study of Torvinen et al. (49), showing no significant changes in the BMC of 56 volunteers (21 men and 35 women; age: 19-38 years) after 8 months of WBV training $\left(1-4 \mathrm{~min} \cdot \mathrm{d}^{-1} ; 3-5 \mathrm{~d} \cdot \mathrm{wk}^{-1} ; 25-45 \mathrm{~Hz}\right)$. The current study used similar values of frequency, amplitude, number of weeks, and daily workload but is not comparable with the duration of the studies mentioned. Similar effects were produced in the study of Milanese et al. (37) after 8 weeks of WBV exercise (2 sessions per week; vibration amplitude $2.0-5.0 \mathrm{~mm}$, vibration frequency $40-60 \mathrm{~Hz}$ ), where was not able to improve bone mineral parameters in young healthy females (mean age: $25.3 \pm 5.26$ years) before the peak bone mass. The positive effect on increasing fat-free tissue by this study allows using this program in other populations such as elderly or sedentary postmenopausal women.

The greatest gains in isokinetic strength occurred at high speeds $\left(270^{\circ} \cdot \mathrm{s}^{-1}\right)$. The effects of WBV training programs are determined by neural adaptation and possible hormonal and biochemical changes. Whole-body vibration exercises may cause excitation of the primary endings of muscle spindles (whose afferent feed-back stimulates increased discharge of $\alpha$-motoneurons) as well as activation of Golgi tendon organs (GTO) that are sensitive to force development and whose activation results in inhibition of muscle action. It can be hypothesized that the cumulative effect of regular systematic WBV training includes (a) enhancement of mono-synaptic stretch-reflexes that are initiated by afferent signals from the muscle spindles to the motoneuron pool and (b) depression of inhibitory impact of GTO due to their accommodation to vibratory-induced excitation (26). The results of this study are consistent with those published by other authors, who indicate that WBV increases the dynamic force of the muscles of the lower extremities $(5,17,27,34,44,48,50)$. Mahieu et al. (34) studied the effect of 6 weeks of WBV in young skiers. The isokinetic strength in the knee extensors improved significantly when compared with baseline. Similar findings were obtained by Delecluse et al. (17) who, after subjecting 74 untrained young women to 12 weeks of WBV, observed that isokinetic strength (at a velocity of $100^{\circ} \cdot \mathrm{s}^{-1}$ ) of the lower extremities was statistically significant improved (17).

There is currently not enough scientific evidence to show that when the vibratory stimulus acts directly on the muscle or tendon occurs tonic vibration reflex occurs, no such evidence exists when the vibration is transmitted to the muscles indirectly (WBV) (27). However, recent research
(45) suggests that when an individual is subjected to WBV on a platform, the body works on a gravitational force that provokes muscles tension. This could explain the strength gains observed in our study. In a review of the effects of vibration on muscle strength, Marin et al. (35) described that greater strength gains are produced with high amplitudes. One possible explanation for the greater results found in the groups that trained with high amplitudes in this study is that working with high amplitude and frequency vibration increases acceleration of the body. However, it has been suggested that WBV training specifically activates type II muscle fibers (43), which were responsible for more explosive movements. The type II muscle fibers activation could explain why the largest increases observed in our study might occur when the force is generated at a high speed $\left(270^{\circ} \cdot \mathrm{s}^{-1}\right)$. One might suggest, but there is insufficient scientific evidence that high amplitude vibration produces greater hypertrophy of type II muscle fibers. Eckhardt et al. (18) in a recent study found that vibration training increased lactate statistically significant compared with exercises performed without vibration, suggesting that this increment could be due to increased recruitment of type II glycolytic fibers during WBV. Based on evidence from this study, WBV can benefit by improving muscle strength in active subjects. In turn, this type of training can supplement athletes by providing assistance to improve their strength and conditioning.

Squat jump and CMJ have yielded no statistically significant differences in any of the variables for any of the jumps made between pretest and posttest. It is possible that the vibration frequency $(50 \mathrm{~Hz})$ coupled with the amplitude $(4 \mathrm{~mm})$ was too strong a stimulus for the groups exposed to the vibration stimulus leading to GTO-mediated reductions in alpha motor neuron firing discharge (31). Similar to this research, de Ruiter et al. (16), evaluated the effects of 11 weeks of WBV static semi-squat on jump height, without obtaining significant improvements. In contrast, other studies assessing the long-term effect of WBV on performance in the vertical jump, both SJ $(14,19)$ as the CMJ $(17,19,44,46,50)$ showed improvements in performance. The differences between our results and those obtained by de Ruiter et al. (16) could be due to the different exercise protocols used. In this study, as in the de Ruiter et al. study (16) participants performed a semi-squat statically, unlike other studies that performed different exercises on the platform dynamically. A possible explanation would be that WBV may increase muscle coactivation (47), and therefore, not increase dynamic actions as vertical jumps (19).

Taking into account that 3 days of training showed improvement when the strength was assessed in this study, we propose to train with 3 sessions per week. Previous research has demonstrated that 4-12 weeks (3 sessions per week) of WBV training can enhance lower-body strength $(17,39)$. Similar results were found by Hong et al. (25), in this study, the WBV group trained along 4-week (3 sessions per week) enhanced neuromuscular activation (RFD). These 
findings suggest that 3 sessions per week WBV training, during at least 4 weeks, are beneficial to increase isokinetic strength at high angular velocities $\left(270^{\circ} \cdot \mathrm{s}^{-1}\right)$.

\section{Practical Applications}

This research shows that using incremental vibratory training, 2 or 3 days per week during 6 weeks of WBV, may increase isokinetic strength and total fat-free mass in recreationally active subjects. However, the improvements are not different between the training groups. G3 produce a significant increase in fat-free-mass when compared with CG. If we use vibrating platforms in an appropriate way, we can have an impact on improving the fitness of this type of subject. Therefore, we consider it a useful complementary tool for personal trainers and coaches when looking for improved fitness. Based on the foregoing information, we propose a number of considerations to take into account in future research. For example, checks to determine whether there are gains of fat-free mass in other segments of the population, using the same protocol as in the current study. Finally, it would be very interesting to compare the static exercise vs. dynamic exercise on the vibration platform to ascertain if the long-term effects involve a loss of coordination in tasks such as jumping, when performing a static-only exercise on the platform. Moreover, further studies are needed to elucidate the exact neurophysiological mechanisms involved in the adaptive responses to vibration exposure in different populations.

\section{ACKNOWLedGments}

The researchers express gratitude to the subjects in this investigation who made this study possible. In addition, the authors acknowledge "UCAM Research Center for High Performance Sport" (Murcia, Spain) for their assistance through-out this project. No funding was received for this article. The results from this study do not constitute endorsement of the products by the authors.

\section{REFERENCES}

1. Alcaraz, PE, Elvira, JL, and Palao, JM. Kinematic, strength, and stiffness adaptations after a short-term sled towing training in athletes. Scand J Med Sci Sports 24: 279-290, 2014.

2. American College of Sports Medicine position stand. Progression models in resistance training for healthy adults. Med Sci Sports Exerc 41: 687-708, 2009.

3. Annino, G, Padua, E, Castagna, C, Di Salvo, V, Minichella, S, Tsarpela, O, Manzi, V, and D'Ottavio, S. Effect of whole body vibration training on lower limb performance in selected high-level ballet students. J Strength Cond Res 21: 1072-1076, 2007.

4. Bojsen-Moller, J, Magnusson, SP, Rasmussen, LR, Kjaer, M, and Aagaard, P. Muscle performance during maximal isometric and dynamic contractions is influenced by the stiffness of the tendinous structures. J Appl Physiol (1985) 99: 986-994, 2005.

5. Bosco, C, Colli, R, Introini, E, Cardinale, M, Tsarpela, O, Madella, A, Tihanyi, J, and Viru, A. Adaptive responses of human skeletal muscle to vibration exposure. Clin Physiol 19: 183-187, 1999.

6. Bosco, C, Iacovelli, M, Tsarpela, O, Cardinale, M, Bonifazi, M, Tihanyi, J, Viru, M, De Lorenzo, A, and Viru, A. Hormonal responses to whole-body vibration in men. Eur J Appl Physiol 81: 449-454, 2000.

7. Brown, L. Isokinetics in Human Performance. Champaign, IL: Human Kinetics, 2000.

8. Candow, DG and Burke, DG. Effect of short-term equal-volume resistance training with different workout frequency on muscle mass and strength in untrained men and women. J Strength Cond Res 21: 204-207, 2007.

9. Cardinale, M. The Effects of Vibration on Human Performance and Hormonal Profile. Budapest, Hungary: Semmelweis University Doctoral School, 2002.

10. Cardinale, $\mathrm{M}$ and Wakeling, J. Whole body vibration exercise: Are vibrations good for you? Br J Sports Med 39: 585-589, 2005.

11. Cardinale, M, Leiper, J, Erskine, J, Milroy, M, and Bell, S. The acute effects of different whole body vibration amplitudes on the endocrine system of young healthy men: A preliminary study. Clin Physiol Funct Imaging 26: 380-384, 2006.

12. Caserotti, P, Aagaard, P, and Puggaard, L. Changes in power and force generation during coupled eccentric-concentric versus concentric muscle contraction with training and aging. Eur J Appl Physiol 103: 151-161, 2008.

13. Caserotti, P, Aagaard, P, Simonsen, EB, and Puggaard, L. Contraction-specific differences in maximal muscle power during stretch-shortening cycle movements in elderly males and females. Eur J Appl Physiol 84: 206-212, 2001.

14. Colson, SS, Pensini, M, Espinosa, J, Garrandes, F, and Legros, P. Whole-body vibration training effects on the physical performance of basketball players. J Strength Cond Res 24: 999-1006, 2010.

15. Da Silva, ME, Fernandez, JM, Castillo, E, Nunez, VM, Vaamonde, DM, Poblador, MS, and Lancho, JL. Influence of vibration training on energy expenditure in active men. $J$ Strength Cond Res 21: 470-475, 2007.

16. de Ruiter, CJ, Van Raak, SM, Schilperoort, JV, Hollander, AP, and de Haan, A. The effects of 11 weeks whole body vibration training on jump height, contractile properties and activation of human knee extensors. Eur J Appl Physiol 90: 595-600, 2003.

17. Delecluse, C, Roelants, M, and Verschueren, S. Strength increase after whole-body vibration compared with resistance training. Med Sci Sports Exerc 35: 1033-1041, 2003.

18. Eckhardt, H, Wollny, R, Muller, H, Bartsch, P, and FriedmannBette, B. Enhanced myofiber recruitment during exhaustive squatting performed as whole-body vibration exercise. J Strength Cond Res 25: 1120-1125, 2011.

19. Fernandez-Rio, J, Terrados, N, Fernandez-Garcia, B, and Suman, OE. Effects of vibration training on force production in female basketball players. J Strength Cond Res 24: 1373-1380, 2010.

20. Fjeldstad, CPI, Bemben, MG, and Bemben, DA. Whole-body vibration augments resistance training effects on body composition in postmenopausal women. Maturitas 20: 79-83, 2009.

21. Gilsanz, V, Wren, TA, Sanchez, M, Dorey, F, Judex, S, and Rubin, C. Low-level, high-frequency mechanical signals enhance musculoskeletal development of young women with low BMD. J Bone Miner Res 21: 1464-1474, 2006.

22. Goto, $\mathrm{K}$ and Takamatsu, K. Hormone and lipolytic responses to whole body vibration in young men. Jpn J Physiol 55: 279-284, 2005.

23. Harrison, AJ and Bourke, G. The effect of resisted sprint training on speed and strength performance in male rugby players. $J$ Strength Cond Res 23: 275-283, 2009.

24. Hazell, TJ, Thomas, GW, Deguire, JR, and Lemon, PW. Vertical whole-body vibration does not increase cardiovascular stress to static semi-squat exercise. Eur J Appl Physiol 104: 903-908, 2008.

25. Hong, JKK, Johnson, ST, and Hoffman, MA. Effects of whole body vibration on electromechanical delay, rate of force development, and presynaptic inhibition. Int J Phys Rehab 1: 30-40, 2010 . 
26. Issurin, VB. Vibrations and their applications in sport. A review. J Sports Med Phys Fitness 45: 324-336, 2005.

27. Jacobs, PL and Burns, P. Acute enhancement of lower-extremity dynamic strength and flexibility with whole-body vibration. $J$ Strength Cond Res 23: 51-57, 2009.

28. Komi, PV. Physiological and biomechanical correlates of muscle function: Effects of muscle structure and stretch-shortening cycle on force and speed. Exerc Sport Sci Rev 12: 81-121, 1984.

29. Kvorning, T, Bagger, M, Caserotti, P, and Madsen, K. Effects of vibration and resistance training on neuromuscular and hormonal measures. Eur J Appl Physiol 96: 615-625, 2006.

30. Lamont, HS, Cramer, JT, Bemben, DA, Shehab, RL, Anderson, MA, and Bemben, MG. Effects of 6 weeks of periodized squat training with or without whole-body vibration on short-term adaptations in jump performance within recreationally resistance trained men. $J$ Strength Cond Res 22: 1882-1893, 2008.

31. Lamont, HS, Cramer, JT, Bemben, DA, Shehab, RL, Anderson, MA, and Bemben, MG. Effects of a 6-week periodized squat training program with or without whole-body vibration on jump height and power output following acute vibration exposure. J Strength Cond Res 23: 2317-2325, 2009.

32. Lamont, HS, Cramer, JT, Bemben, DA, Shehab, RL, Anderson, MA, and Bemben, MG. Effects of adding whole body vibration to squat training on isometric force/time characteristics. $J$ Strength Cond Res 24: 171-183, 2010.

33. Ligouri, GC, Shoepe, TC, and Almstedt, HC. Whole body vibration training is osteogenic at the spine in college-age men and women. J Hum Kinet 31: 55-68, 2012.

34. Mahieu, NN, Witvrouw, E, Van de Voorde, D, Michilsens, D, Arbyn, V, and Van den Broecke, W. Improving strength and postural control in young skiers: Whole-body vibration versus equivalent resistance training. J Athl Train 41: 286-293, 2006.

35. Marin, PJ and Rhea, MR. Effects of vibration training on muscle strength: A meta-analysis. J Strength Cond Res 24: 548-556, 2010.

36. Martinez-Pardo, E, Romero-Arenas, S, and Alcaraz, PE. Effects of different amplitudes (high vs. low) of whole-body vibration training in active adults. J Strength Cond Res 27: 1798-1806, 2013

37. Milanese, CPF, Simoni, C, and Zancanaro, C. Mild chronic whole body vibration does not affect bone mineral mass or density in young females. J Hum Sport Exerc 6: 474-479, 2011.

38. Rauch, F, Sievanen, H, Boonen, S, Cardinale, M, Degens, H, Felsenberg, D, Roth, J, Schoenau, E, Verschueren, S, and Rittweger, J. Reporting whole-body vibration intervention studies: Recommendations of the international society of musculoskeletal and neuronal interactions. J Musculoskelet Neuronal Interact 10: 193-198, 2010.

39. Reyes, GF, Dickin, DC, Crusat, NJ, and Dolny, DG. Whole-body vibration effects on the muscle activity of upper and lower body muscles during the baseball swing in recreational baseball hitters. Sports Biomech 10: 280-293, 2011.

40. Rhea, MR, Alvar, BA, Burkett, LN, and Ball, SD. A meta-analysis to determine the dose response for strength development. $\mathrm{Med} S \mathrm{Sc}$ Sports Exerc 35: 456-464, 2003.
41. Rhea, MR, Bunker, D, Marin, PJ, and Lunt, K. Effect of iTonic whole-body vibration on delayed-onset muscle soreness among untrained individuals. J Strength Cond Res 23: 1677-1682, 2009.

42. Rittweger, J. Vibration as an exercise modality: How it may work, and what its potential might be. Eur J Appl Physiol 108: 877-904, 2010.

43. Rittweger, J, Beller, G, and Felsenberg, D. Acute physiological effects of exhaustive whole-body vibration exercise in man. Clin Physiol 20: 134-142, 2000.

44. Roelants, M, Delecluse, C, Goris, M, and Verschueren, S. Effects of 24 weeks of whole body vibration training on body composition and muscle strength in untrained females. Int J Sports Med 25: $1-5,2004$.

45. Roelants, M, Verschueren, SM, Delecluse, C, Levin, O, and Stijnen, V. Whole-body-vibration-induced increase in leg muscle activity during different squat exercises. $J$ Strength Cond Res 20: 124-129, 2006.

46. Ronnestad, BR. Comparing the performance-enhancing effects of squats on a vibration platform with conventional squats in recreationally resistance-trained men. $J$ Strength Cond Res 18 : 839-845, 2004.

47. Rothmuller, C and Cafarelli, E. Effect of vibration on antagonist muscle coactivation during progressive fatigue in humans. $J$ Physiol 485(pt 3): 857-864, 1995

48. Torvinen, S, Kannu, P, Sievanen, H, Jarvinen, TA, Pasanen, M, Kontulainen, S, Jarvinen, TL, Jarvinen, M, Oja, P, and Vuori, I. Effect of a vibration exposure on muscular performance and body balance. Randomized cross-over study. Clin Physiol Funct Imaging 22: 145-152, 2002.

49. Torvinen, S, Kannus, P, Sievanen, H, Jarvinen, TA, Pasanen, M, Kontulainen, S, Nenonen, A, Jarvinen, TL, Paakkala, T, Jarvinen, M, and Vuori, I. Effect of 8-month vertical whole body vibration on bone, muscle performance, and body balance: A randomized controlled study. J Bone Miner Res 18: 876-884, 2003.

50. Verschueren, SM, Roelants, M, Delecluse, C, Swinnen, S, Vanderschueren, D, and Boonen, S. Effect of 6-month whole body vibration training on hip density, muscle strength, and postural control in postmenopausal women: A randomized controlled pilot study. J Bone Miner Res 19: 352-359, 2004.

51. Viru, A. Plasma hormones and physical exercise. Int J Sports Med 13: 201-209, 1992.

52. Vissers, D, Verrijken, A, Mertens, I, Van Gils, C, Van de Sompel, A, Truijen, S, and Van Gaal, L. Effect of long-term whole body vibration training on visceral adipose tissue: A preliminary report. Obes Facts 3: 93-100, 2010

53. Wilcock, IM, Whatman, C, Harris, N, and Keogh, JW. Vibration training: Could it enhance the strength, power, or speed of athletes? $J$ Strength Cond Res 23: 593-603, 2009. 